\title{
Conseils médicaux aux fumeurs et fumeuses
}

\author{
Nicolas Broccard ${ }^{a}$, Anne-Katharina Burkhalter ${ }^{b}$ \\ a Journaliste scientifique, Berne; ${ }^{b}$ Programme national d'arrêt du tabagisme, responsable du projet Vivre sans tabac, Berne
}

\begin{abstract}
La troisième édition du document "Conseil médical aux fumeurs et fumeuses» comporte un schéma de conseil remanié et d'autres nouveautés. L'accent repose sur la pratique en cabinet. Le manuel est disponible dès à présent en allemand et en français.
\end{abstract}

\section{Deux offres pour arrêter de fumer}

Pour la première fois, la Journée stop-tabac aura lieu le 31 mai 2016 à l'occasion de la Journée mondiale sans tabac. Le concours pour arrêter de fumer débutera le lendemain. Les deux événements sont organisés par le Programme national d'arrêt du tabagisme.

\section{Journée stop-tabac}

Les fumeuses et les fumeurs peuvent essayer de se passer de cigarettes pendant une journée. Cent bons d'une valeur de 25 francs chacun seront tirés au sort parmi toutes les participantes et tous les participants inscrits sur www.stop-tabac-31mai.ch.

\section{Concours pour arrêter de fumer}

Toutes les personnes qui réussissent à s'abstenir de fumer entre le $1^{\text {er }}$ et le 30 juin peuvent prendre part au tirage au sort doté de 100 prix (20 fois 500 francs et 80 fois 50 francs en espèces). Possibilité de s'inscrire en ligne à l'adresse www.stop-tabac-31mai.ch ou en renvoyant la carte d'inscription.

\section{Matériel}

La carte d'inscription, une affiche et d'autres documents peuvent être commandés gratuitement à l'adresse www.stop-tabac31mai.ch.

Organisations partenaires: Fédération des médecins suisses (FMH), Société suisse des pharmaciens (pharmaSuisse), Société suisse des médecins-dentistes (SSO), Association suisse des infirmiers et infirmières (ASI) et Swiss Dental Hygienists. La Journée stop-tabac et le concours pour arrêter de fumer sont financés par le fonds de prévention du tabagisme.

tion pour le cabinet. Projet

Vivre sans tabac,

Programme national

d'arrêt du tabagisme,

Berne 2015 (l'ouvrage peut être commandé auprès de Mme Anne-Katharina Burkhalter).

Correspondance: Anne-Katharina Burkhalte Programme national d'arrêt du tabagisme Projet Vivre sans tabac

Haslerstrasse 30 CH-3008 Berne

Tél. 0315991020 Fax 0315991035 annekburkhalter[at] freivontabak.ch

de 2009 reposait encore sur le «modèle transthéo rique» (Stages of Change Model) et en particulier sur les phases de contemplation, de préparation et d'action. Une méta-analyse réalisée par Cochrane en novembre 2010 a toutefois mis en évidence que ce modèle n'était pas plus efficace que d'autres en ce qui concerne les conseils lors de l'arrêt du tabagisme

\section{Les conseils en cinq étapes}

La nouvelle édition du document "Conseil médical aux fumeurs et fumeuses» [1] se base sur le modèle des cinq A en se référant explicitement à la technique de l'entretien de motivation. Le schéma de conseil est structuré comme suit:

- Ask: identifier le tabagisme.

- Assess: évaluer la motivation à arrêter de fumer et la dépendance à la nicotine.

- Advise: recommander fermement l'arrêt du tabagisme à tous les fumeurs et fumeuses, même ceux qui ne sont pas motivés.

- Assist: en fonction du degré de motivation, prévoir une brève consultation ou un entretien de motivation pour discuter de l'arrêt du tabagisme ou planifier une intervention plus complète.

- Arrange: aborder dans tous les cas le tabagisme lors de la prochaine consultation et directement fixer d'autres rendez-vous lorsque le fumeur décide de cesser sa consommation.

Les autres recommandations de la nouvelle édition concernent principalement les points suivants:

- Les chapitres concernant l'efficacité de la varénicline et du bupropion ont été mis à jour, de même que les informations relatives à la prise en charge des frais de traitement par l'assurance-maladie obligatoire.

- Les différents traitements pharmacologiques combinés avec substitut nicotinique sont présentés en détail.

- La cigarette électronique est présentée en détail, et les avantages de son utilisation en vue du sevrage tabagique font l'objet d'un résumé en huit recommandations.
- La diminution de la consommation de cigarettes est traitée dans un chapitre séparé.

Par ailleurs, un nouveau chapitre est dédié au sevrage tabagique dans des conditions-cadres et des groupes de population spécifiques: jeunes, diminution de la consommation de cigarettes, femmes, grossesse et allaitement, patientes et patients hospitalisés ou ayant subi une opération, dépendance à l'alcool, consommation de cannabis, dépression, schizophrénie et groupes de population défavorisés. 